\title{
Exploración posturográfica de pacientes simuladores
}

Jorge Rey-Martínez, Julio Rama-López y Nicolás Pérez-Fernández

Departamento de Otorrinolaringología. Clínica Universitaria. Facultad de Medicina. Universidad de Navarra. Pamplona. Navarra. España.

Introducción: El patrón afisiológico en la posturografía dinámica computarizada (PDC) puede observarse en el contexto de muy diversas etiologías. Analizamos un grupo de pacientes simuladores según los resultados obtenidos en esta prueba.

Pacientes y método: En este trabajo analizamos particularmente un grupo de 7 pacientes simuladores, esto es los que, sin que se conozca a priori la ganancia esperada de un informe patológico, muestran una alteración del equilibrio falsa e irreal que sólo se reconoce como "inestabilidad continua" y genera una alteración postural compleja. Analizamos los registros obtenidos en sus PDC según los criterios diagnósticos descritos por otros autores.

Resultados: De los criterios analizados, encontramos que el descrito por Cevette et al es el más frecuentemente positivo en nuestro grupo de estudio.

Conclusiones: La diversidad de criterios utilizados hace necesario utilizarlos todos en conjunto, partiendo de un alto índice de sospecha y mucha cautela en el diagnóstico.

Palabras clave: Simulador. Inestabilidad. Posturografía. Vestibular.

\section{Posturographic Examination of Malingering Patients}

Introduction: An aphysiological pattern in computerized dynamic posturography (CDP) may be produced by numerous causes. We analyze the results obtained in this test by a group of malingering patients.

Patients and method: This study analyzes a particular group of 7 malingerers, ie patients simulating a false and unreal equilibrium disorder, without any a priori awareness of the gain expected from a pathological report. The condition can only be identified with the very complex postural disorder known as "continuous imbalance." We analyze the results of the dynamic posturography test following the diagnostic criteria described by other authors.

Results: Of the criteria analyzed, we found Cevette's to be the most frequently positive for our study group.

Conclusions: The wide diversity in the criteria used makes it necessary to apply them together, assuming a high level of suspicion and great care in the diagnostic process.

Key words: Malingering. Imbalance. Posturography. Vestibular.

\section{INTRODUCCIÓN}

El patrón afisiológico en posturografía dinámica fue descrito inicialmente por Hamid ${ }^{1}$. Para su definición es necesario que se cumplan los siguientes criterios:

1. Puntuación baja en condiciones sencillas.

Este artículo es sobre un trabajo de investigación que fue galardonado en el Congreso Nacional de la SEORL de 2005.

Los autores no manifiestan ningún conflicto de intereses.

Correspondencia: Dr. J. Rey.

Departamento de ORL. Clínica Universitaria.

Facultad de Medicina. Universidad de Navarra.

Avda. Pío XII, 36. 31008 Pamplona. Navarra. España.

Correo electrónico: jrey@unav.es

Recibido el 7-10-2005.

Aceptado para su publicación el 13-3-2007.
2. Cambios significativos del patrón de déficit sensorial al repetir la prueba de organización sensorial (SOT).

3. Control voluntario de la postura en términos de mejora de balanceo y aumento de la latencia en respuesta a perturbaciones de la superficie de apoyo y/o el entorno visual.

Posteriormente, Cevette et $\mathrm{al}^{2}$ establecieron una fórmula que permite diferenciar fácilmente los patrones "normal", "vestibular" y "afisiológico" aplicando una fórmula matemática a los resultados que el paciente obtuvo en el SOT. Esto ha facilitado mucho el diagnóstico, pues resulta ser una de las medidas más fáciles de estudiar. Desde entonces, numerosos estudios han intentado resolver el dilema de este diagnóstico y han aparecido diversos criterios para definir de manera más precisa y objetiva este patrón.

En general, el patrón afisiológico aparece en pacientes simuladores con trastornos somatomorfos (como reacción de conversión, somatización) y en la enfermedad depresiva y los trastornos de ansiedad. 
El objetivo de este trabajo es analizar una pequeña población de pacientes catalogados como simuladores y estudiar en ellos los diversos criterios que definen el patrón afisilógico.

\section{PACIENTES Y MÉTODO}

En este trabajo se revisarán los resultados del estudio postural de 7 pacientes vistos en nuestra consulta por presentar diversos grados de inestabilidad, pero que en el seguimiento, en algún caso prolongado durante 1 año, fueron diagnosticados como simuladores.

El objetivo de este trabajo es analizar una pequeña población de pacientes catalogados como simuladores y estudiar en ellos los diversos criterios que definen el patrón afisilógico.

Estos pacientes buscaban algún tipo de compensación (laboral, económica y/o social) manifestando una sensación de inestabilidad crónica. En casi todos ellos hubo un evento inicial de tipo vestibular (vértigo y/o mareo) de causas variadas (traumática en 3 casos, idiopática en 2 y tras neuritis vestibular en 2). En el estudio inicial y el seguimiento prolongado se ha podido detectar una fuerte disparidad clinicoexploratoria, con una variabilidad muy importante en los resultados y ausencia de respuesta a todas las modalidades terapéuticas indicadas. En todos los casos, después de un cuidadoso seguimiento en el que se involucró a los médicos de sus empresas y/o casas aseguradoras, se pudo reconocer este hallazgo como secundario a la búsqueda de una compensación muy particular.

\section{Exploración otoneurológica}

En todos los casos se realizó un estudio completo audiovestibular, que incluía otoscopia, exploración de nistagmo espontáneo (con y sin fijación de la mirada), nistagmo posicional, maniobra oculocefálica, nistagmo de agitación cefálica, exploración de movimientos y seguimiento ocular, videonistagmografía, posturografía dinámica computarizada (PDC) y audiometría tonal liminar. Esta exploración otoneurológica se repitió en más de una ocasión en algún paciente. En todos los pacientes se solicitó interconsulta con el departamento de neurología para descartar cualquier tipo de alteración central.

\section{Posturografía dinámica computarizada}

Para este estudio empleamos el equipo de PDC Smart Equitest System versión 7.0 (Neurocom International Inc., Clackamas, Estados Unidos). El sistema está conformado por un soporte informático, una plataforma móvil referida al movimiento que experimenta el propio paciente durante la exploración y un entorno visual.

La PDC se basa en la detección del desplazamiento del centro de presión corporal, casi siempre fiel reflejo del centro de gravedad, en diferentes situaciones de conflicto sensorial (visuosomatosensorial) o estimulación vestibular.

Se realiza a cada paciente un SOT, una prueba de control motor (MCT) y una prueba de adaptación (AT).

El resultado de cada prueba se clasifica de acuerdo a los siguientes criterios:
SOT. En cada condición se determina el grado de estabilidad, que se calcula comparando la diferencia angular entre la máxima oscilación anteroposterior del paciente durante cada prueba y los límites máximos de estabilidad anteroposterior normal. Se obtiene así, en cada una de ellas, una puntuación de equilibrio (puntuación de equilibrio SOT, 1-6), que se basa en la hipótesis de que un individuo puede realizar un desplazamiento anteroposterior sin caerse de $12,5^{\circ}$ alrededor de un eje situado en los tobillos. La puntuación se calcula comparando el desplazamiento actual máximo del centro de gravedad y ese teórico máximo, y se ofrece como porcentaje entre el $0 \mathrm{y}$ el $100 \%$, representando el primero un desplazamiento máximo y, por tanto, la necesidad de dar un paso en la plataforma para evitar caerse y el segundo, un balanceo mínimo o nulo con una estabilidad perfecta. El SOT se representa mediante: el porcentaje de equilibrio, el análisis sensorial o contribución relativa de los receptores somatosensoriales, visuales y vestibulares en la estabilidad global del paciente, así como su capacidad para mantener el equilibrio con informaciones sensoriales erróneas y el análisis de la estrategia de respuesta.

MCT. Se estudian las respuestas automáticas de control postural del paciente frente a ciertas perturbaciones de la superficie de apoyo. Las medidas realizadas son: simetría de distribución de masa, velocidad de reacción o latencia y fuerza y simetría de la respuesta. Para ello se emplean seis estímulos. En tres, la plataforma se desplaza desde el centro hacia delante y en los otros tres, desde el centro hacia atrás. Cada uno de estos movimientos se realiza a diferentes intensidades, corto, medio y largo (small, medium y large respectivamente), definidos por la amplitud del movimiento y la velocidad de desplazamiento.

$P A$. Se realizan dos tipos de movimientos con la plataforma, hacia arriba y hacia abajo, siguiendo el eje del tobillo. Cada una de las dos condiciones se repite en cinco ocasiones, la repetición se realiza siempre con los mismos parámetros de tiempo (400 ms) y amplitud de movimiento $\left(8^{\circ}\right)$. El sentido de esta prueba es objetivar el grado de adaptación o mejoría del paciente a medida que se repite la prueba en las mismas condiciones (disminución gradual del balanceo a expensas de la estrategia de tobillo).

\section{Criterios de patrón afisiológico}

De la revisión bibliográfica extraemos los siguientes criterios que vamos a analizar en nuestros pacientes (ReyMartínez JA, Rama-López J. Exploración posturográfica de pacientes simuladores. Pamplona: Clínica Universitaria de Navarra. Universidad de Navarra; 2005. Disponible en: http://www.aeternum.tv/vertigo).

Criterio 1 (Cevette). Una estrategia es "afisiológica" cuando el paciente se comporta posturalmente mejor en las condiciones más difíciles, con mayor conflicto sensorial, que en las sencillas y con menor conflicto sensorial ${ }^{2}$. Esto es, los resultados son mejores en el SOT 5 y el SOT 6 que en los SOT 1-4. 


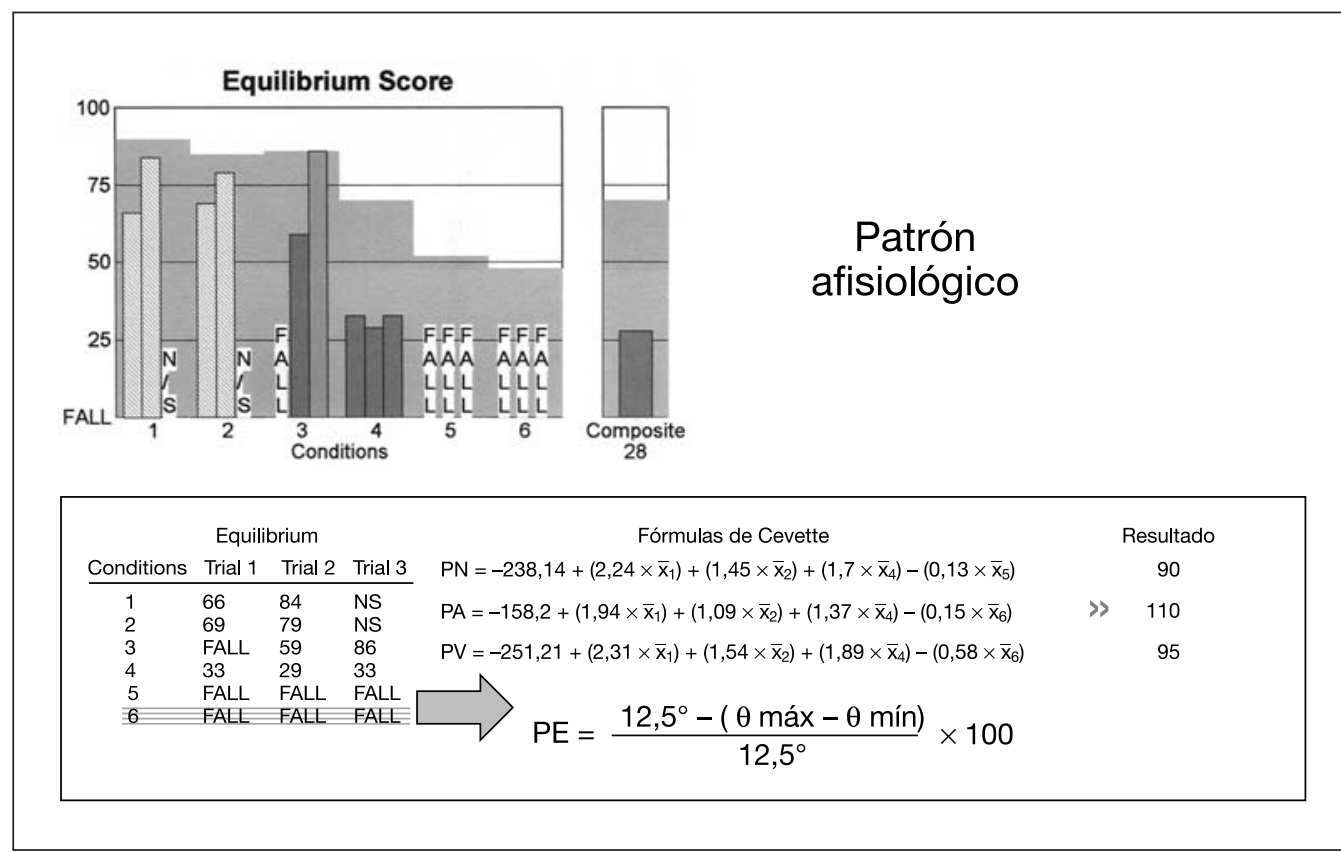

Figura 1. Metodología para obtener las puntuaciones de Cevette. Partiendo del valor medio de los intentos de cada condición, se aplican las fórmulas de Cevette, y el patrón obtenido es el de mayor puntuación. En este caso, el patrón afisiológico. PA: patrón afisiológico; PE: puntuación de equilibrio; PN: patrón normal; $P V$ : patrón vestibular.

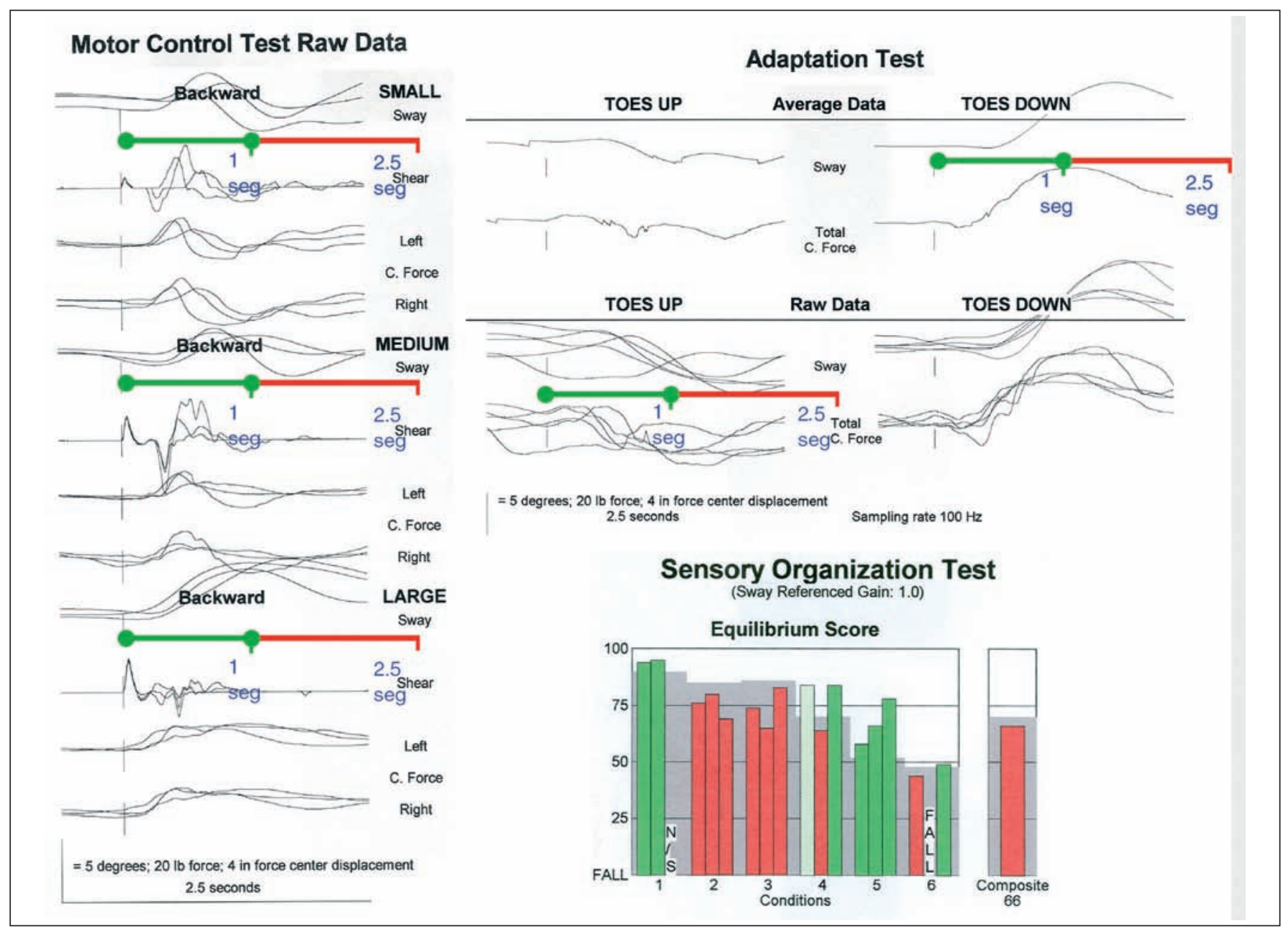

Figura 2. En este caso llama la atención la prolongación del balanceo en el test de adaptación, aunque también cumple el criterio en el test de control motor. Este caso cumplía también el criterio 1 (mayor puntuación en condiciones más complejas). Se ha añadido barras de tiempo a las gráficas de los tests de adaptación y de control motor; en su primer tramo se encuentra el primer segundo de la prueba; según estos criterios, lo que queda hacia la derecha se considera afisiológico, pues tiene una duración excesiva. 


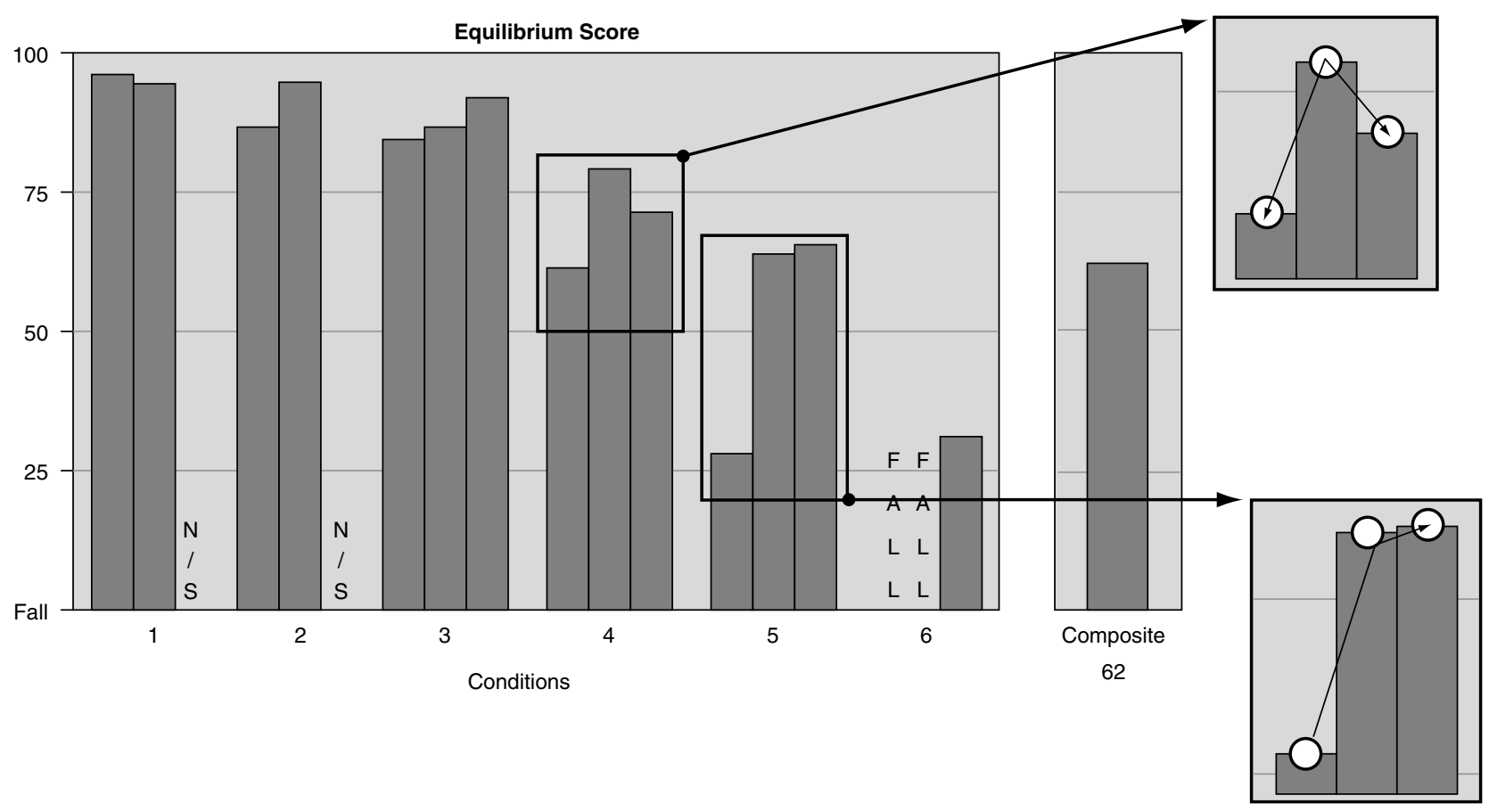

Figura 3. Paciente diagnosticado de enfermedad de Ménière. En la condición 4 se observa una variabilidad menor y en la condición 5 , un fenómeno claro de adaptación rápida.

Criterio 2 (Cevette). En los pacientes con patrón afisiológico hay una gran variabilidad entre los intentos, lo que conforma una respuesta "errática"2. En cada condición de la prueba de organización sensorial se realizan tres intentos; lo normal es que la respuesta sea idéntica en cada intento o que mejore en cada repetición (adaptación). La respuesta errática es fácil de reconocer por lo variado que resulta cada resultado en la misma condición (fig. 1). Este fenómeno es más fácilmente reconocible en la MCT y en la de PA.

Criterio 3. Puntuación según Cevette et $\mathrm{al}^{2}$. Estos autores definieron tres fórmulas, cada una de las cuales determina los patrones normal, afisiológico y vestibular. Estas fórmulas son las siguientes:

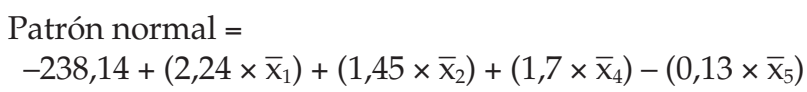

Patrón normal $=$$$
-238,14+\left(2,24 \times \overline{\mathrm{X}}_{1}\right)+\left(1,45 \times \overline{\mathrm{x}}_{2}\right)+\left(1,7 \times \overline{\mathrm{X}}_{4}\right)-\left(0,13 \times \overline{\mathrm{x}}_{5}\right)
$$

Patrón afisiológico $=$

$$
-158,2+\left(1,94 \times \overline{\mathrm{x}}_{1}\right)+\left(1,09 \times \overline{\mathrm{x}}_{2}\right)+\left(1,37 \times \overline{\mathrm{x}}_{4}\right)-\left(0,15 \times \overline{\mathrm{x}}_{6}\right)
$$

\section{Patrón vestibular $=$}

$$
-251,21+\left(2,31 \times \overline{\mathrm{X}}_{1}\right)+\left(1,54 \times \overline{\mathrm{x}}_{2}\right)+\left(1,89 \times \overline{\mathrm{X}}_{4}\right)-\left(0,58 \times \overline{\mathrm{x}}_{6}\right)
$$

A partir de los resultados obtenidos en cada paciente se obtiene el valor adecuado y el patrón definitivo es el que obtiene mayor valor (fig. 2).
Criterio 4. Según Goebel et $\mathrm{al}^{3}$, a partir de un análisis multifactorial, se define el patrón afisiológico de la combinación de los siguientes resultados: resultado patológico en SOT1, respuesta exagerada en MCT con estímulo small y respuesta inconsistente al obtener una respuesta idéntica en MCT con estímulo small y large o incluso mayor en el primero que en la segunda.

Criterio 5 (Allum et al ${ }^{4}$ ). En la MCT y/o la PA, pasado $1 \mathrm{~s}$ del estímulo hay una respuesta exagerada y desmesurada. Ese período pasado el estímulo pertenece al dominio del control voluntario de la estabilidad y refleja una desestabilización exagerada (fig. 3).

Criterio 6 (Shepard $\left.{ }^{5}\right)$. Disparidad clinicoexploratoria. El paciente con un patrón posturográfico muy deteriorado es capaz de desplazarse, caminar, posicionarse en el equipo, colaborar en la colocación del arnés, etc., lo que manifiesta un control postural y de la marcha normal, no relacionado con la respuesta tan deteriorada que luego manifiesta. En ocasiones la disparidad es tan evidente que ésta se hace evidente al ver otras partes de la exploración, como el estudio de los límites de estabilidad.

Criterio $7\left(\right.$ Shepard $\left.^{5}\right)$. La aleatorización o repetición de la prueba da unos resultados diferentes, no repetibles. Cuando se repite el SOT o la MCT, cambiando el orden de pre- 
sentación de condiciones y de estímulo, el paciente presenta resultados diferentes de los iniciales.

\section{RESULTADOS}

De la población de pacientes que formaban parte de nuestro estudio, el 57,1\% eran varones, con una media \pm desviación estándar de edad de $39 \pm 7$ (34-55) años.

Entre los resultados obtenidos de la aplicación de los criterios a nuestros pacientes, destacan como los que más veces han dado positivas: el criterio 3 (puntuación de Cevette et al), con un $88,8 \%$ de verdaderos positivos (los resultados de la aplicación de las fórmulas de Cevette en nuestra población se detallan en la tabla I), y el criterio 5 (tiempo de reacción voluntario) con un $100 \%$ de verdaderos positivos, si bien este criterio no se pudo valorar en la primera de las pruebas que se realizó a los pacientes con pruebas repetidas en el tiempo del estudio (pacientes 3 y 7 ).
En cuanto al criterio que menos veces fue positivo en nuestra casuística, fue el criterio 1 (las puntuaciones del SOT no empeoran en condiciones teóricamente más complejas), con un $90 \%$ de falsos negativos.

En nuestro estudio, el máximo de criterios que son positivos en un paciente los presentan los pacientes 5 y 7 , que cumplen $4 / 7$ criterios. El paciente 1 es el que menos criterios cumple, únicamente 2 . Finalmente, el número de criterios positivos que más frecuentemente presentan los pacientes (moda) es $3(57,1 \%)$. La relación completa de criterios aplicados en cada paciente se muestran en la tabla II.

\section{DISCUSIÓN}

Podemos decir que evidentemente no hay un criterio mejor o único que nos permita definir el patrón afisiológico y que es necesario considerarlos todos. Éste debe partir de una sospecha clínica fehaciente que tenga en consideración

Tabla I. Puntuaciones obtenidas por cada paciente en la escala de Cevette

\begin{tabular}{|c|c|c|c|c|c|c|c|c|c|}
\hline Paciente & Media 1 & Media 2 & Media 3 & Media 4 & Media 5 & Media 6 & $P N$ & $P A$ & $P V$ \\
\hline 2 & 82,3 & 79 & 46,3 & 0 & 0 & 0 & 60,8 & 87,6 & 60,6 \\
\hline $3(1)$ & 84,5 & 61,6 & 0 & 0 & 0 & 0 & 40,5 & 72,9 & 38,8 \\
\hline $3(2)$ & 58,6 & 50 & 27 & 0 & 0 & 0 & $-34,4$ & 10 & $-38,8$ \\
\hline 4 & 24,3 & 0 & 2,3 & 0 & 0 & 0 & -184 & -111 & -195 \\
\hline $5(2)$ & 66,3 & 0 & 57,6 & 0 & 0 & 0 & $-89,6$ & $-29,6$ & $-98,1$ \\
\hline 6 & 90,3 & 0 & 86 & 0 & 0 & 0 & $-35,9$ & 17 & $-42,6$ \\
\hline $7(1)$ & 94,5 & 75 & 74 & 77,3 & 67,3 & 31 & 205 & 208,1 & 210,7 \\
\hline $7(2)$ & 17 & 0 & 0 & 0 & 0 & 0 & -200 & -125 & -212 \\
\hline
\end{tabular}

PA: patrón afisiológico; PV: patrón vestibular. Media es el valor medio obtenido en la puntuación de la prueba de organización sensorial (SOT) en cada condición. El 1 o el 2 entre paréntesis junto al número del paciente identifican la repetición de las pruebas posturográficas ordenadas en el tiempo.

Tabla II. Resultados de los pacientes para cada criterio

\begin{tabular}{|c|c|c|c|c|c|c|c|}
\hline Paciente & Criterio 1 & Criterio 2 & Criterio 3 & Criterio 4 & Criterio 5 & Criterio 6 & Criterio 7 \\
\hline 1 & No & No & $A$ & No & Sí & NV & NV \\
\hline 2 & No & Sí & $A$ & No & Sí & NV & NV \\
\hline $3(2)$ & No & No & $A$ & No & Sí & NV & Sí \\
\hline 4 & No & Sí & $A$ & Sí & Sí & NV & NV \\
\hline $5(2)$ & No & No & $A$ & Sí & Sí & Sí & No \\
\hline 6 & No & No & A & No & Sí & NV & NV \\
\hline $7(1)$ & Sí & No & V & NV & NV & No & Sí \\
\hline $7(2)$ & No & No & $A$ & Sí & Sí & No & Sí \\
\hline
\end{tabular}

A: patrón afisiológico; No: no cumple el criterio; NV: criterio no valorable; Sí: cumple el criterio; V: patrón vestibular. El 1 o el 2 entre paréntesis junto al número del paciente identifican la repetición de las pruebas posturográficas ordenadas en el tiempo. 
la exploración dinámica de la marcha y la postura. Resultaría un error tomar algún hallazgo particular, inexplicable, como definitivo y establecer un diagnóstico de este patrón, con lo que esto implica.

Si bien en este trabajo hemos seleccionado a pacientes muy particulares, tampoco podemos olvidar que los pacientes con enfermedades psiquiátricas concretas desarrollan también patrones de este tipo ${ }^{6}$.

En cuanto a lo revisado respecto a los criterios de otros autores, creemos que la formulación de Cevette es la más adecuada. Resulta fácil de realizar y es objetiva. En nuestra casuística podemos decir que se cumple en todos los sujetos. En el paciente número 7 no es así en la primera exploración, pero sí en la segunda. Al contrario que el paciente número 5, el número 7 muestra una gran variabilidad entre criterios.

Estamos de acuerdo, y nuestro estudio así lo confirma, en que probablemente, más que el SOT, la MCT y la PA son las más adecuadas ${ }^{1}$. En nuestra casuística lo cumplen todos los sujetos.

Los otros criterios posturográficos se presentan en nuestros pacientes con menor frecuencia. Además, no hemos encontrado ninguna asociación cuando se cumple el resto de los criterios que nos permiten establecer un patrón de utilidad diagnóstica para ayudarnos a valorar a estos pacientes, en el caso de que alguna de las otras pruebas fuera positiva.

Por lo tanto, para definir un patrón posturográfico como afisiológico es necesario:
- Valorar adecuadamente de forma clínica el comportamiento postural del paciente.

- Observar que hay disparidad en su deambulación normal con la realización de la MCT y la PA.

- Aplicar en casos sospechosos la fórmula de Cevette.

- Apreciar la disfunción de este patrón en las alteraciones de el SOT que, en cierta manera, reflejan una inadecuada, por ilógica, utilización de aferencias sensoriales. La complejidad del fenómeno cognitivo en el control postural hace que esta prueba sea muy difícil de valorar.

\section{BIBLIOGRAFÍA}

1. Hamid MA. New test of vestibular function. En: Meyers EN, Bluestone CD, editores. Advances in Otolaryngology-Head and Neck Surgery. Vol. 5. Chicago: Year Book Medical; 1990. p. 15-38.

2. Cevette MI, Puetz B, Marion MS, Wertz ML, Muenter MD. Aphysiologic performance on dynamic posturography. Otolaryngol Head Neck Surg. 1995;112:676-88.

3. Goebel JA, Sataloff RT, Hanson JM, et al. Posturographic evidence of nonorganic sway patterns in normal subjects, patients and suspected maligners. Otolaryngol Head Neck Surg. 1997;117:293-302.

4. Allum JH, Shepard NT. An overview of the clinical use of dynamic posturography in the differential diagnosis of balance disorders. J Vestib Res. 1999;9:223-52.

5. Shepard NT. Vestibular Rehabilitation Programs. En: Shepard NT, Steven A, editores. Practical management of the balance disorder patient. Singular; 1996. p. 184.

6. Pérez N, Pla J. Cinetosis y vértigo psicógeno. En: Bartual J, Pérez N, editores. El sistema vestibular y sus alteraciones. Vol. II. Barcelona: Masson; 1999. p. 459-68. 